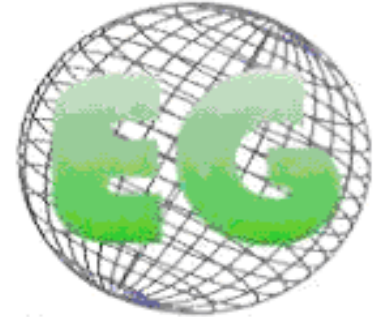

ISSN 1695-6141

$\mathrm{N}^{\circ} 21$

\title{
PROPUESTA PRÁCTICA ANTE LA DISCAPACIDAD. EL USO DEL TAI CHI Y DEL QIGONG.
}

PRACTICAL PROPOSAL TO COMBAT DISCAPACITY. THE USE OF TAI CHI AND QIGONG.

\author{
*Carrillo Vigueras, J., **Gómez López, M., ***Vílchez Conesa, P. \\ *Hospital General Universitario Reina Sofía. **Facultad de Ciencias del Deporte. ***Grupo de Investigación \\ “ Actividad Físico-Deportiva y Calidad de Vida” . Universidad de Murcia.
}

Palabras clave: Tai Chi, Autocuidado, Movilidad reducida, Qigong, Calidad de vida

Keywords: Tai Chi, Qigong, self-care, quality of life.

\section{RESUMEN}

En el presente trabajo se valoran las posibilidades del Qigong en personas con movilidad reducida en los miembros inferiores, con el objetivo fundamental de mejorar la calidad de vida de estas personas. Presentamos una propuesta práctica formada por ejercicios sencillos, que justifica y adapta el Qigong a una población especial, como lesionados medulares, parapléjicos, hemipléjicos o cualquier patología limitadora de la marcha, ya sea en el hogar o en el hospital. La instrucción en el autocuidado es la parte esencial de este trabajo con personas adultas. Los resultados obtenidos en las diferentes experiencias que se han realizado han supuesto un fortalecimiento muscular y sobre todo un cambio en la actitud y calidad de vida de las personas.

\section{ABSTRACT}

In the present study, we evaluate the possibilities of Qigong exercise in people with low leg mobility, with the ultimate aim of improving their quality of life. We present a practical proposal which consists of easy exercises that justifies and adapts Qigong to a special population such as injured core, paraplegic, hemiplegic or any other condition limiting walking at home or in the hospital. The instruction in self care is the most important aspect in this work with adult people. The results obtained from the different experiments are an increase in muscle strength and above all change of attitude and quality of life for those affected. 


\section{INTRODUCCIÓN}

La relación entre ejercicio físico y salud ha sido objeto de innumerables estudios epidemiológicos que coinciden en aseverar que es un elemento condicionante de la calidad de vida [1], de la salud y del bienestar [2, 3], que produce efectos beneficiosos tanto físicos como psicológicos en las personas $[4,5,6,7,8,9,10,11,12,13,14]$ asociándose a un menor riesgo de mortalidad y morbilidad. Los cuidados de las personas deben partir desde los profesionales de la salud ayudando a éstas a proporcionarse un autocuidado de calidad. Los estudios sugieren que las personas físicamente activas suelen tener dos o tres años más de expectativas de vida [15].

El Tai Chi es un ejercicio físico tradicional que proviene de China. Se basa en una serie de movimientos armónicos unidos entre ellos con una secuencia continua en la que el cuerpo está constantemente cambiando de posición, con un centro de gravedad más bajo. Durante la ejecución del Tai Chi, se requiere una respiración profunda y una concentración mental para alcanzar la armonía entre el cuerpo y la mente. El Qigong consiste en una serie de movimientos estructurados que unidos, usualmente, a una respiración abdominal proporcionan los mismos beneficios que el Tai Chi, siendo el desplazamiento de piernas y pies muy reducido.

Muchos estudios transversales sugieren que la práctica regular del Tai Chi es beneficiosa para la salud, ya que mejora el sistema cardiorrespiratorio, la flexibilidad, la movilidad articular, la fuerza muscular o la composición corporal [16] y la salud mental, reduciendo el nivel de estrés [17].

Otros de los beneficios que tiene el Tai Chi, es el control postural y la reducción del riesgo de caídas [18, 16], lo que cobra mayor importancia cuando se habla de poblaciones especiales que dependen del control del tronco y de la propiocepción para mantener su postura en sedentación y en las actividades de la vida diaria. Según Petridou et al. [19], las caídas y las contusiones son las lesiones más comunes entre los niños discapacitados, mientras que las lesiones en las extremidades superiores y las lesiones por sobreesfuerzo son las menos comunes.

Lamentablemente, existen poblaciones que no son capaces de desarrollar un programa de Tai Chi tradicional, debido a su falta de movilidad, la cual se relaciona con un peor estado de salud. De hecho, hay estudios que relacionan el tiempo de sedentación con una peor percepción de la salud [20]. En estos sujetos, en los que no podemos variar la posibilidad de sedentación, se necesitan buscar alternativas para mejorar su calidad de vida. En esta línea, Chen et al. [21] adaptaron un estilo de Tai Chi simplificado y Qigong en mayores con enfermedades crónicas y largas estancias en residencias u hospitales, obteniendo resultados positivos.

Wall [17] utilizó el Tai Chi en niños en edad escolar con el fin de reducir el estrés. Los ejercicios se llevaron a cabo en sillas, manteniendo el raquis alineado durante la sedentación. En esta posición, mejoró la fisiología de la respiración, como la perfusión y la respuesta relajante que daba lugar a una cascada de beneficios para el sistema inmunitario y el humor.

Los discapacitados, permanentes o temporales, con movilidad reducida en los miembros inferiores pueden obtener los mismos beneficios del Tai Chi que los pacientes de artritis reumatoide, como son el descenso del dolor articular, la menor fatiga, el mayor rango de 
movimiento o la mejora de la calidad de vida, de ahí que esta actividad física esté apoyada por la Fundación Australiana de Artritis [18].

Por lo tanto, son muchos los estudios que demuestran que esta modalidad de actividad física es totalmente adaptable a las personas con discapacidad y a pacientes con enfermedades crónicas [16]. Es importante, dada la falta de movilidad en esta población, llevar a cabo una fisioterapia respiratoria. Chang et al. [22] comprobaron que un programa de Tai Chi y Qigong durante 12 semanas mejora la función pulmonar incluso en poblaciones con dificultades respiratorias como los asmáticos.

Los agentes de salud, pueden y debe influir sobre la actividad de las personas y ayudar a reformar el estilo de vida de los individuos para mejorar su salud. Desde la perspectiva enfermera el uso de los patrones funcionales de Gordon [23] nos va a proporcionar una visión holística. Este describe dichos patrones como una configuración de comportamientos, más o menos comunes a todos los individuos que contribuyen a su salud, calidad de vida y al logro de su potencial humano, y que se dan de una manera secuencial a lo largo del tiempo. La valoración usando los Patrones Funcionales permite de una manera sistematizada obtener los datos suficientes para explicar el modelo que se propone desde esta perspectiva.

La modalidad que proponemos de Tai Chi podría clasificarse como una actividad de fuerza de baja intensidad y un entrenamiento de la estabilidad y el balance articular [24], que recoge todos los beneficios ya mencionados para esta población.

\section{PROPUESTA PRÁCTICA DE BĀ DUÀN JINN (八段锦) SENTADO}

La serie de Bā duàn Jinn (八段锦) es una forma de Qigong marcadamente terapéutica pues se aconseja su realización para prevenir todo tipo de enfermedades, ya sea en la posición de pie como en la de sentado [25]. Esta serie de ocho ejercicios (Bā duàn) se utiliza para favorecer una mejor calidad de vida, mayor longevidad y mejorar, a la vez que proporcionar estabilidad, flexibilidad, tonicidad en músculos y todos los beneficios que llevan asociados tanto el Qigong como el Tai Chi [13]. Los sistemas terapéuticos tradicionales se dirigen a las personas, no a sus males [26].

Para la realización de esta serie de ejercicios, se parte desde la posición de sentado, aflojándose la ropa y sentándose sobre una superficie estable, sobre el suelo con una toalla doblada, una manta o una colchoneta enrollada. Colocándonos con las piernas cruzadas, la mujer con la pierna izquierda por fuera, el hombre con la pierna derecha por fuera [27]. Las manos se pueden colocar de dos maneras diferentes: por un lado haciendo un mudra, los extremos de los dedos pulgar y medio (corazón) de una mano se unen cogiendo el pulgar de la otra mano entre ellos, mientras que ese pulgar presiona, en la mano, entre las primeras falanges de los dedos índice y medio (en un punto de acupuntura llamado Lao Kong), por fuera el resto de dedos de esa mano cubren a los de la otra (forma de Wudang) (figura 1); por otro lado sin hacer el mudra, una mano conteniendo a la otra. Siguiendo los cánones taoístas, la mujer debe unir los dedos de la mano izquierda y el hombre los de la mano derecha. Las manos descansan sobre el abdomen, cerca del ombligo. 


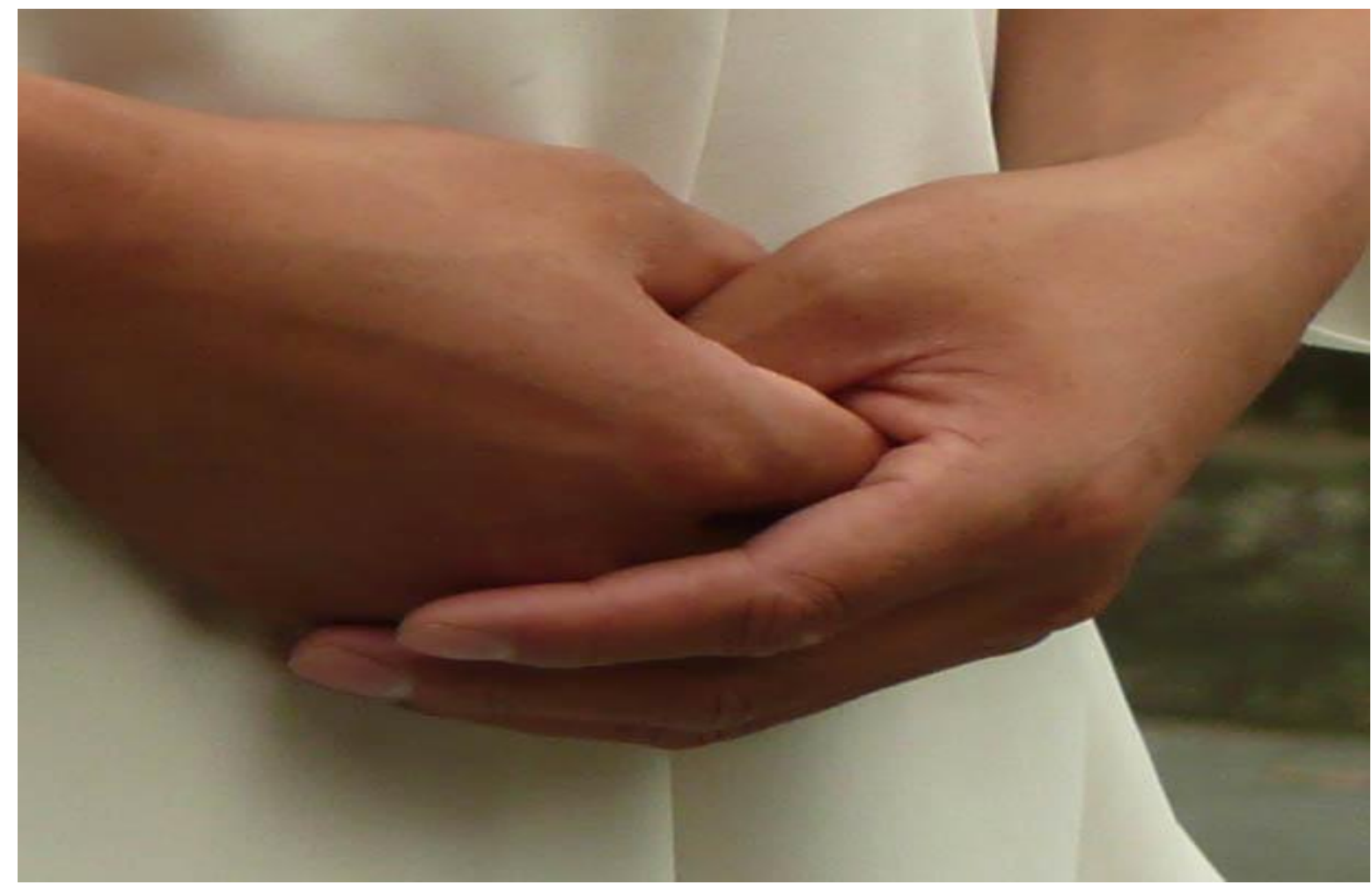

Figura 1. Posición de las manos

En esta posición, es preciso cerrar los ojos e intentar vaciar la mente de cualquier pensamiento que la distraiga ya que mantener los ojos así favorece que la atención no se disperse y se mantiene aquietado el Shén (espíritu). Según Aparicio [28] el cuerpo es una manifestación más burda de la energía (moléculas, materia), y el Shén (así denominan al conjunto de todas las actividades mentales) una manifestación más depurada y sutil de la energía básica. Se debe fijar la atención al principio en la respiración y enfocar la mente en la temperatura que tiene el aire al entrar en los pulmones, sintiéndolo en la nariz frío y cargado de oxígeno y notarlo salir caliente y viciado, con poca energía útil para el cuerpo. La mente ha de estar tranquila, vacía.

Practicar esta forma sentados es fundamental para que la sensación de enraizamiento nos proporcione un aspecto sólido y firme a partir de esta posición [29]. La práctica de esta forma en el desarrollo de la energía es fundamental. Con esta postura se ha de llegar a una meditación igual o más profunda aún que desde la posición de meditación de pie (Wu chi), al trabajar la energía de una forma más sutil. La práctica regular y adecuada proporciona numerosos beneficios tanto físicos como psíquicos, por no mencionar los espirituales.

Los ejercicios en esta posición favorecen la circulación energética de los meridianos de energía (meridianos de acupuntura) de la parte superior del cuerpo sobre todo los que transcurren por los brazos. Es aconsejable hacer este tipo de ejercicios por la mañana al levantarse con efectos especiales en personas que están encamadas o con dificultades para mantenerse de pie [29].

Es beneficioso permanecer en esta posición un tiempo suficiente para apaciguar la mente, insistiendo al principio especialmente en la relajación para que paulatinamente se vaya introduciendo la respiración como elemento básico del movimiento. 
La espalda ha de estar recta, los hombros relajados y sueltos al igual que la cintura pélvica.

Primer Duan (primera serie): Sostener el monte Kunlun con ambas manos.

Partiendo desde la posición sentada, se obliga a que los dientes castañeteen de manera suave pero audible durante 36 veces [26, 29, 30] o 35 veces [29], la punta de la lengua está en el paladar. Este ejercicio estimula las glándulas salivares, y al estimularse éstas, se produce una activación del sistema parasimpático provocando, desde el inicio de la serie, que el cuerpo esté cada vez más relajado y tranquilo. A su vez favorece que las piezas dentarias se fijen con mayor dureza a las mandíbulas por un aumento del riego sanguíneo. Tras el castañeteo se relaja la mandíbula un momento y cuando la boca se llene de saliva hay que tragarla en tres degluciones.

Posteriormente hay que llevar las palmas de las manos con los dedos entrelazados y los codos abiertos hasta cubrir las orejas respirando suavemente durante 9 ciclos respiratorios (figura 2).

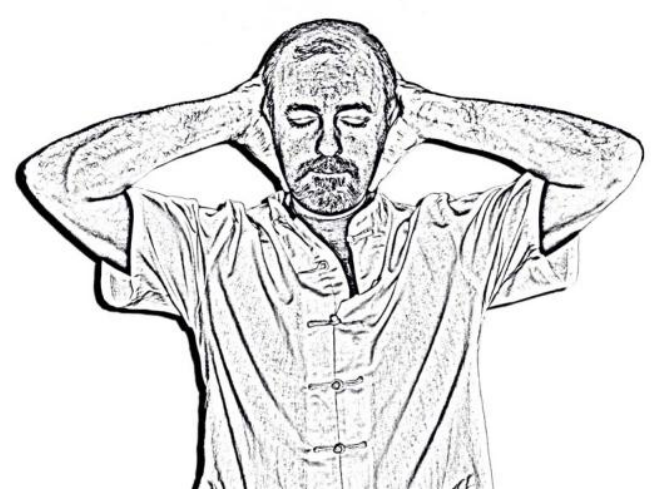

Figura 2. Palmas de las manos, dedos entrelazados y codos abiertos

Serrato [27] y González [32] proponen una flexión del tronco hacia adelante a la vez que se cierran suavemente los codos en una espiración y se vuelve a inspirar abriendo los codos y estirando el cuerpo en una inspiración se repite el ciclo en doce ocasiones. Los demás autores no indican movimiento alguno en esta posición. Considerar su inclusión puede tener efectos beneficiosos, pero también conlleva un cierto grado de dificultad añadida que habría que valorar dependiendo de la condición física del practicante.

Zhong Li, citado por Shixin [30], el gran teórico de la dinastía Tang (618-907), establece que tras llevar las manos a las orejas con los dedos entrelazados, éstas se depositen sobre las orejas abriendo los dedos en abanico colocando el dedo índice sobre el dedo corazón y golpeando de esta manera en ambos lados de la cabeza y en la zona occipital durante 24 veces. Se acaba con una respiración profunda inhalando y exhalando lentamente. Sin embargo Navarrete [29] propone que el golpeteo se haga por encima de la zona de la oreja y no por detrás.

Este ejercicio alivia, pero sobre todo previene los dolores de cabeza, los mareos y de manera sustancial la rigidez de cuello al producirse un desbloqueo de los meridianos que recorren la zona: vaso gobernador, meridiano de vejiga y meridiano de vesícula biliar. 
Una vez finalizadas las repeticiones, las manos vuelven con las palmas hacia arriba hasta depositarse encima de las rodillas y se vuelve a hacer una respiración profunda durante nueve veces. Posteriormente se retorna a la meditación.

Segundo Duan: Agitar o vibrar el tronco o pilar celeste.

Partiendo del final de la serie anterior, sentado con la espalda recta y relajada, los hombros igualmente relajados y las manos sobre las rodillas, se llevan estas últimas frente al ombligo. La palma de la mano derecha contiene la palma de la mano izquierda con los dedos ligeramente doblados y se rota suavemente la cabeza primero hacia un lado y después hacia el otro [30]. La mirada se dirige ligeramente hacia arriba en ambos lados durante unos dos segundos. Zhengcai [31] propone elevar los hombros para tocar con ellos el mentón en su giro hacia cada lado, al igual que Serrato [27].

Cada uno de los movimientos se ha de realizar con doce repeticiones a cada lado, un total de veinticuatro. El tronco en todo momento ha de estar erguido (figura 3).

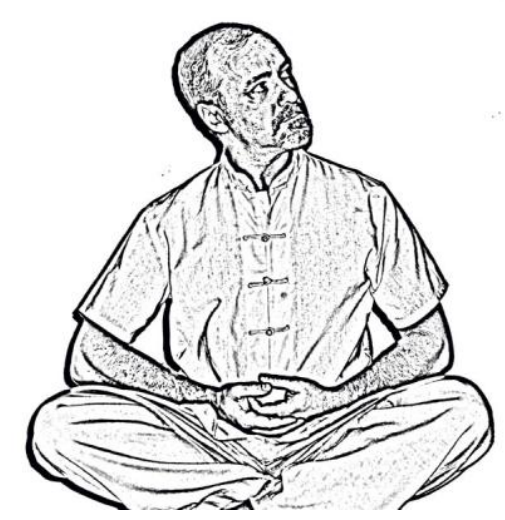

Figura 3. Tronco erguido

La respiración se hace exhalando al ir hacia atrás e inhalando al regresar al centro y la atención ha de ir siempre en la respiración. Las variaciones de esta serie se realizan con las manos en la nuca o bien en las rodillas. El movimiento ha de ser lento y pausado como la respiración.

Se moviliza la energía del cuello, de las vértebras cervicales y de la base del cráneo por donde discurren siete grandes meridianos. En ningún momento se ha de comprimir la nuca o realizar movimientos bruscos, en el caso de que exista alguna tensión o contractura en el cuello, es aconsejable no realizar esta forma.

Se consigue una gran flexibilidad en los hombros y en la nuca. Con esta pieza, la rotación se transmite hasta la altura de las vértebras lumbares y hace que la energía que circula por los meridianos de la espalda se equilibre previniendo enfermedades y aliviando a las que ya están instauradas. Se fortalece la parte anterior del tórax y ayuda al alivio de las tensiones emocionales. 
Tercer Duan: Elevar los brazos, gargarismos con saliva o acumular el chi.

Se parte de la posición de sentado, con la espalda y los hombros relajados. Elevamos los brazos con los puños cerrados por encima de la cabeza como para agarrar una barra sobre ella (figura 4).

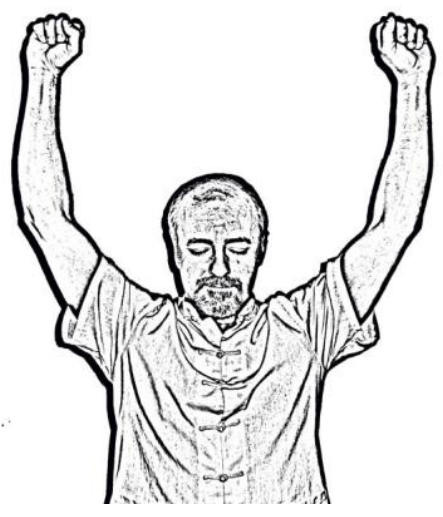

Figura 4. Elevación de los brazos con puños cerrados

Es necesario apretar con la lengua el paladar duro desde delante hacia atrás, hacia el paladar blando (úvula o campanilla) con la punta de la lengua para producir saliva [29]. Shixin [30] propone que se mueva la lengua por toda la boca unas 36 veces para producir saliva, en cambio Serrato [27] propone que se hagan en 36 ocasiones con la lengua hacia la derecha y otras tantas hacia la izquierda. Por último todos coinciden en que se ha de tragar la saliva en tres tragos produciendo ruido al hacerlo.

La producción de saliva es muy valorada de manera terapéutica, puesto que la secreción de este producto con buena calidad es esencial en un proceso digestivo óptimo. La producción de saliva implica que una vez más el sistema nervioso autónomo parasimpático está funcionando correctamente y las glándulas asociadas trabajan de igual manera. La calidad del citado fluido corporal puede cambiar dependiendo de las emociones (secreción de cortisol en caso de estrés) haciendo que se fluidifique o bien que se espese dependiendo, entre otras, de las comidas a las que nos enfrentamos 0 a las emociones en las que vivimos. De ahí la importancia que reviste esta forma.

Las manos en lo alto permiten que el acto de la deglución de la saliva se haga de forma correcta, por lo que es esencial esta pieza cuando las personas están encamadas y tienen dificultad en la deglución, o bien hay algún problema de regurgitación o hernia de hiato. Mejora los problemas de periodontitis y de encías a nivel de la boca.

Al finalizar la deglución de la saliva las manos vuelven de forma natural sobre las rodillas o bien se llevan al Dantien (bajo el ombligo) para acumular la energía que ha bajado con la saliva [31].

Las tres primeras Duan o series forman un esquema tipo Yang es decir actúan sobre problemas y situaciones de la parte frontal del cuerpo. 
Las tres formas o Duan siguientes son de tipo Ying ya que se trabaja principalmente sobre la espalda, la región lumbar y los hombros.

Cuarto Duan: Masaje en la región lumbar.

Partiendo desde la posición de sentado con la espalda bien recta pero relajada, nos frotamos las manos hasta calentarlas considerablemente y damos un masaje a la región lumbar durante unas treinta y seis vueltas (figura 5). Hacemos un círculo hacia adentro bajando por el interior de la columna y subiendo por la parte externa [33]. De esta forma favorecemos la circulación sanguínea de la zona y su elasticidad. Se hace descender el fuego del corazón hasta los riñones donde reside la energía (se provoca una hiperemia lumbar bilateral).

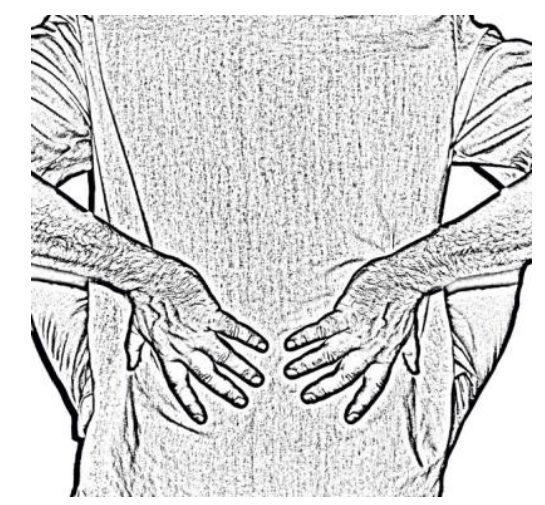

Figura 5. Masaje en la región lumbar - Cuarto Duan

Serrato [27] propone un cierre fin de pieza subiendo los brazos lateralmente con las palmas hacia arriba hasta colocarlas sobre la cabeza en el punto de las mil reuniones, Bai Hui (punto más alto de la cabeza). Todo esto debe realizarse en inspiración e iniciar la exhalación bajando los brazos con las palmas hacia abajo por delante del cuerpo hasta dejarlas en el Dantien.

Al terminar, regresamos las manos al frente y las relajamos apoyándolas sobre las rodillas.

Quinto Duan: Rotar una mano en la cintura o girar el torso hacia un lado.

Desde la finalización de la posición anterior, una mano se lleva hacia la espalda, sin girar, y la palma de la otra hacia el ombligo. Se pasa desde una posición Yin a una Yang la sensación ha de ser como de estar girado. La tensión generada a nivel lumbar se utiliza para transmitirla a las vértebras del lado retorcido y el movimiento del lateral contrario para ajustar la posición de éstas. La mirada hacia adelante se fija en un punto distante.

Se rota un hombro hacia adelante y el otro en sentido contrario, treinta y seis veces con el dorso de la mano derecha en la región lumbar derecha y otras tantas cambiando de mano y de zona lumbar con la mano izquierda (dorso). Se lleva a la cabeza en suave movimiento con el hombro (figura 6). Los primeros ejercicios que se hacen es normal que se oigan los huesos acomodarse puesto que se realiza a una liberación de la tensión, razón por la que 
hay que hacerlo con suavidad [29]. Hacer todas las repeticiones de un lado y después cambiar al otro.

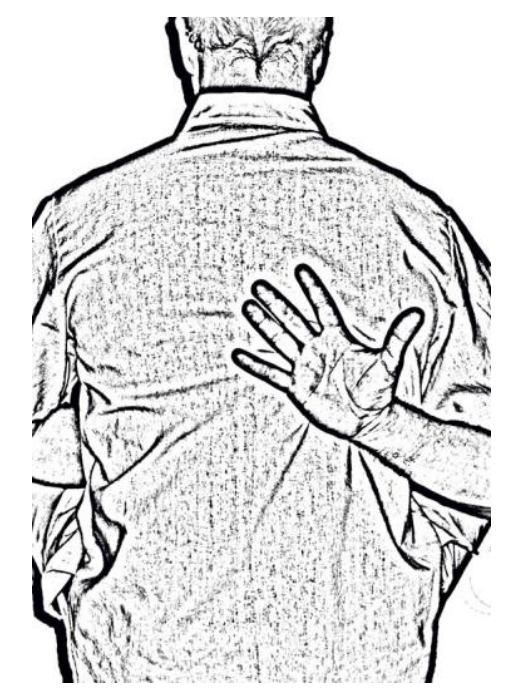

Figura 6. Suave movimiento con el hombro

El beneficio de esta serie se va a comprobar en una mayor elasticidad de toda la espalda y hombros, un mayor ajuste de las vértebras y una corrección en movimiento de los huesos del tórax.

Sexto Duan: Rotando las manos en la cintura.

Navarrete [29] por su parte sostiene que partiendo desde la posición de sentados se colocan las palmas de las manos en la región lumbar correspondiente y se giran los hombros haciendo círculos hacia delante con un hombro y hacia atrás con el otro durante 36 molinetes (figura 7). Por otra parte, Shixin [30] propone el movimiento de los hombros de igual manera y coloca las manos en el Dantien, bajo el vientre la mano derecha conteniendo la izquierda para las mujeres y la mano izquierda conteniendo la derecha para los hombres. Serrato [27] en cambio introduce un movimiento que no se describe en los anteriores que consiste en masajear el Dantien durante 36 veces o más en sentido de las agujas del reloj para las mujeres y en sentido contrario para los hombres, con un inicio subiendo palmas hacia arriba hasta la altura del pecho y bajando tras un giro de éstas hasta el Dantien. La realización de cualquiera de las tres variantes es válida por su efecto terapéutico y el realizarlo de una u otra manera no conlleva perjuicio alguno. 


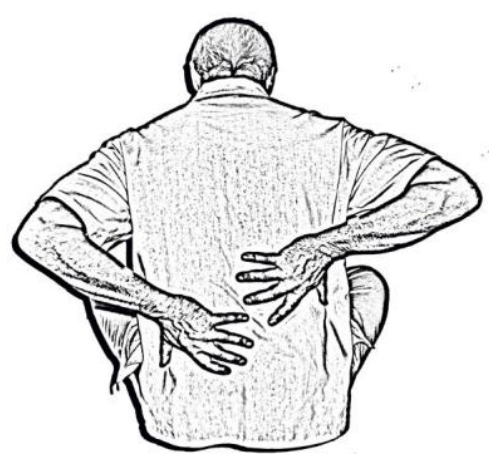

Figura 7. Palmas de la mano en la región lumbar

Tras estos movimientos se termina estirando las piernas y dejándolas relajadas con los dedos de los pies apuntando hacia arriba, la espalda recta y los hombros relajados. Zhengcai [31] propone, una vez estiradas las piernas, mover los brazos de una forma diferente, ligeramente flexionados se hacen círculos con un hombro primero y con el otro después, contando 36 vueltas con cada uno. Prouzet [33] señala que el giro de los brazos es con ellos estirados, los dos a la vez van de atrás hacia adelante en la vertical y viceversa en ambos casos 36 veces.

Habitualmente se genera un calor interno que es normal y puede suceder que se llegue incluso a sudar produciéndose algún escalofrío. Al estirar las piernas se libera toda la tensión que se acumulaba y se produce una respiración tranquila y fluida y una sensación de plenitud y de fortalecimiento del cuerpo.

Las formas finales son, al igual que las formas de pie, una síntesis del trabajo realizado y en ellas se integran de forma clara la respiración y la elasticidad.

Séptimo Duan: Sujetar el cielo con los dedos entrelazados.

Se vuelven a cruzar las piernas como se describe en la posición de sentados y se sitúan las manos a la altura del Dantien, con las palmas hacia arriba entrelazando los dedos y presionando con los dedos meñiques el abdomen. Navarrete [29] plantea que se calienten las manos con el aliento durante unas cuantas respiraciones. Se elevan las manos hacia el pecho y se sigue subiendo girando suavemente las manos a la altura de la cara para ponerlas con las palmas hacia arriba y los dorsos hacia abajo como si sujetaran algo pesado. Se hace una inspiración en este movimiento. Zhengcai [31] indica que hay que hacer este movimiento con las piernas estiradas (figura 8). 


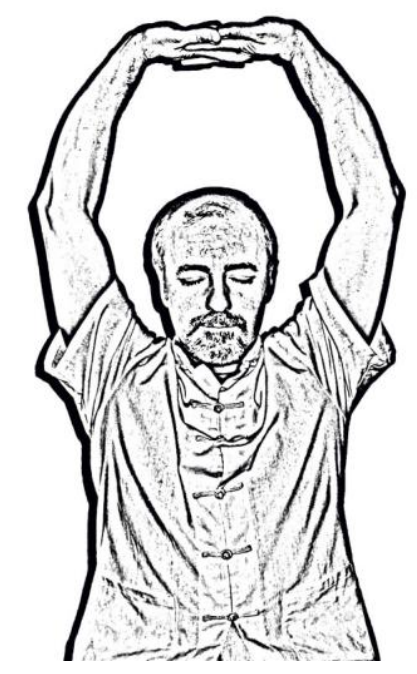

Figura 8. Estiramiento de brazos con las piernas estiradas

Tras este movimiento, Serrato [27] y Zhengcai [31] plantean que se inicie la exhalación, se suelten los dedos y se bajen las manos rotándolas para colocarlas sobre la cabeza una sobre la otra, la derecha por fuera los hombres y la izquierda por fuera las mujeres, se dan tres golpes suaves con las manos y se realizan varias respiraciones completas.

Después, exhalando se bajan las manos con las palmas hacia abajo y con los dedos enfrentados hasta el Dantien para repetirlo durante nueve veces. No se debe agachar la cabeza en ningún momento. Se debe seguir con los ojos y con la mirada el recorrido de las manos para optimizar el trabajo realizado.

Con esta serie se trabajan energéticamente los meridianos de los brazos favoreciendo la circulación de la energía, de la sangre haciendo que la respiración sea más profunda al elevar la caja torácica, mientras se desciende el diafragma.

Octavo Duan: Tirarse de los dedos de ambos pies o estirar las piernas.

Hay que estirar las piernas y colocarlas juntas ayudándose de las manos que mantienen la postura de sentados al apoyarse en el suelo con los dedos mirando hacia los pies, calentar las manos si se precisa, bajando desde la cadera procurando no doblar demasiado la espalda ni elevar las rodillas y por último masajear los pies (figura 9).

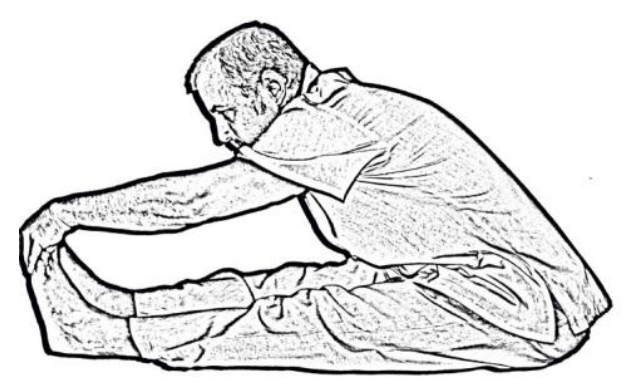

Figura 9. No doblar demasiado la espalda ni elevar las rodillas 
Alcanzar la planta de los pies requerirá su tiempo pero poco a poco haciendo los ejercicios se consigue estirar y flexibilizar los músculos para conseguir el objetivo marcado. La forma se ha de realizar aunque no se consiga llegar a tocar la planta del pie la consecución de este objetivo puede llevar unos tres meses, pero se logra con una buena relajación.

El masaje de los pies se hace tanto por las plantas como por el dorso tocando todos los puntos sensibles y favoreciendo de esta manera el retorno venoso y la estimulación de los meridianos. Primero se hace con las dos manos en un pie y más tarde se pasa al otro. El movimiento se ha de repetir durante 12 ocasiones de un lado a otro estirando y aflojando las piernas para aumentar progresivamente la elasticidad.

Tras estos movimientos se vuelve a la posición de sentado con las piernas cruzadas o estiradas (como mejor se alcance la concentración). Es necesario estimular una vez más la producción de saliva en la boca girando la lengua en su interior, para tragarla en tres sonoras degluciones. Las manos las situamos frente al Dantien haciendo un mudra con los extremos de los dedos pulgar y medio (corazón) de una mano se unen cogiendo el pulgar de la otra mano entre ellos mientras que ese pulgar presiona entre las primeras falanges de los dedos índice y corazón (en un punto de acupuntura llamado Lao Kong). Por fuera, el resto de dedos de esa mano cubren a los de la otra.

Desde esta posición se puede rotar la cabeza hacia la derecha y hacia la izquierda, siguiendo los hombros ligeramente el movimiento de la cabeza durante unos 24 movimientos.

Llega el momento de descansar en esta posición con las manos situadas sobre las rodillas, con los ojos cerrados y sintiendo cómo la energía fluye libremente por su cuerpo.

Para terminar uno se levanta lentamente y realiza una pequeña caminata pisando con fuerza, para que la circulación sanguínea se restablezca en las piernas y se equilibre la circulación de energética.

\section{CONCLUSIÓN}

El uso de técnicas de autocuidado como Taichi y Qigong por personas con movilidad reducida es sin lugar a dudas un importante avance dentro de su discapacidad. El reiniciar el movimiento, por pequeño que sea, supone abrir una luz a la recuperación funcional del sistema músculo-esquelético. La ejecución de estos sencillos movimientos que se proponen suponen un fortalecimiento muscular y sobre todo un cambio actitudinal favorecido por la utilización diferente de los músculos respiratorios.

El uso de técnicas y ejercicios sencillos para que los pacientes encamados puedan reiniciar su movilidad es un recurso más a aplicar por los especialistas de los cuidados que desde la Atención Primaria visitan a este tipo de pacientes en sus hogares. Una sencilla explicación puede rentabilizar el tiempo empleado y acortar el periodo de cama que un paciente necesita para su recuperación funcional.

El uso mínimo de instalaciones y de materiales lo hacen especialmente económico, economía que se ve favorecida por no necesitar de un grupo determinado para la práctica pudiendo realizarse con una sola persona o con más. La instrucción clara y progresiva hace que el valor añadido obtenido sobre la salud se multiplique. 


\section{REFERENCIAS}

[1] Biddle, S. Psychological benefits of exercise and physical activity. Revista de Psicología del Deporte 1993; 4: 99-107.

[2] Blasco, T. Actividad física y salud. Barcelona: Martínez Roca; 1994.

[3] Corbin, CB., Pangrazi, RP. \& Welk, GJ. Towards an understanding of appropriate physical activity levels for youth. Physical Activity and Fitness Research Digest 1994; 1: 1-8.

[4] American College of Sports Medicine Position stand: Exercise and physical activity for older adults. Medicine and Science in Sports and Exercise 1998; 30: 992-1008.

[5] Barriopedro, MI., Eraña, I. \& Mallol, L. Relación de la actividad física con la depresión y satisfacción con la vida en la tercera edad. Revista de Psicología del Deporte 2001; 10: $239-246$.

[6] Bennett, J., Carmack, MA. \& Gardner, VJ. The effect of a program of physical exercise on depression in older adults. Physiology Educational 1982; 39: 21-24.

[7] Casterad, JC., Serra, JR. \& Beltrán, M. Efectos de un programa de actividad física sobre los parámetros cardiovasculares en una población de la tercera edad. Apunts. Educación física y deportes 2003; 73: 42-48.

[8] De Gracia, M. \& Marcó, M. Efectos psicológicos de la actividad física en personas mayores. Psicothema 2000; 12: 285-292.

[9] Eriksen, G. Physical fitness and changes in mortality: the survival of the fittest. Sports Medicine 2001; 31: 571-576.

[10] Matsudo, S. \& Matsudo, V. Prescription and benefits of physical activity in the third age. Revista Brasileira de Ciencia \& Movimiento 1992; 6: 19-30.

[11] Matsudo, S., Matsudo, V. \& Neto, T. Efeitos benéficos da actividade física na aptidao física e saúde mental durante o processo de envelhecimento. Revista Brasileira de Actividade Física \& Saúde 2000; 5: 60-76.

[12] Myers, J., Kaykha, A., George, S., Abella, J., Zaheer, N., Lear,S., Yamakazi, T. \& Froelicher, V. Fitness versus physical activity patterns in predicting mortality in men. American Journal of Medicine 2004; 117: 912-918.

[13] Taylor-Piliae, R. \& Froelicher, ES. Effectiveness of Tai Chi exercise in improving aerobic capacity: a meta-analysis. The Journal of Cardiovascular Nursing 2004; 19: 48-57.

[14] Torrado, VM., Aparici, M. \& Sanz, P. Efectos psicológicos de un programa de entrenamiento físico en sujetos mayores de 60 años. Revista Española de Geriatría y Gerontología 1994; 29: 138-142.

[15] Paffenbarger, RS. \& Lee, IM. Physical activity and fitness for health and longevity. Research Quarterly for Exercise and Sport 1996; 67 (Supplement): 11-28.

[16] Wong, AM., Lin, Y-C., Chou, S-W., Tang, F-T. \& Wong, P-Y. Coordination Exercise and Postural Stability in Elderly People: Effect of Tai Chi Chuan. Arcivesh Physical Medicine Rehabilitation 2001; 82: 608-12.

[17] Wall, RB. Tai Chi and Mindfulness-Based Stress Reduction in a Boston Public Middle School. Journal of Pediatric Health Care 2005; 19: 230-237.

[18] Lee, MS., Pittler, MH. \& Ernst, E. Tai chi for rheumatoid arthritis: systematic review. Rheumatology 2007; 46: 1648-1651.

[19] Petridou, E., Kedikoglou, S., Andrie, E., Farmakakis, T., Tsiga, A., Angelopoulos, M. et al. Injuries among disabled children: a study from Greece. Injury Prevention 2003; 9: 226-230.

[20] Garatachea, N., Molinero, O., Martínez, R., Jiménez, R., González, J. \& Márquez, S. Feelings of well being in elderly people: Relationship to physical activity and physical function. Archives of Gerontology and Geriatric 2009; 48: 306-312.

[21] Chen, K-M., Lin, K-M., Lin, H-S., Wu, H-C., Chen, W-T., Li, C-H. et al. The effects of a Simplified Tai-Chi Exercise Program (STEP) on the physical health of older adults 
living in long-term care facilities: A single group design with multiple time points. International Journal of Nursing Studies 2008; 45: 501-507.

[22] Chang, Y-F., Yang, Y-H., Chen, C-C. \& Chiang, B-L. Tai Chi Chuan training improves the pulmonary function of asthmatic children. Journal of Microbiology and Immunology Infect 2008; 41: 88-95.

[23] Gordon, M. (2007). Manual de diagnósticos de Enfermería. Mcgraw-Hill Interamericana de España.

[24] Judge, JO. Balance Training to Maintain Mobility and Prevent Disability. American Journal of Preventive Medicine 2003; 25(3): 150-156.

[25] Carrillo, J. Técnicas de afrontamiento del estrés: Tai Chi. Murcia: SATSE; 2008.

[26] Aparicio, AJ. La antropología aplicada, la medicina tradicional y los sistemas de cuidado natural de la salud: una ayuda intercultural para los padecimientos crónicos. Gazeta de Antropología 2007; 23: 14. [On line] [Disponible en http://www.ugr.es/ pwlac/G23_14AlfonsoJulio_Aparicio_Mena.html] [Consulta 2009, $15,03]$.

[27] Serrato, JC. Las Baduan Jin en posición de sentado [On line] 2009 [Disponible en http://www.wudao.es/La_Baduan_jing_sentado4.php] [Consulta 2009, 15, 03].

[28] Aparicio, AJ. Idea de salúd intercultural. Una aproximación antropológica a la idea de salud intercultural, derivada de la medicina tradicional china, en contacto con diferentes culturas. Gazeta de Antropología 2004; 20: 20-05.

[29] Navarrete, A. El brocado de las Trece Posiciones del QiGong en Qigong Association of América. [On line] $2006 \quad$ [Disponible http://www.qi.org/articles/el_brocado_de_las_trece_posicion.htm] [Consulta 2009, 03, 15].

[30] Shixin, L. Baduanjin "Brocade" Exercices in eight forms. [On line] 1997 [Disponible en http://www.shme.com/kungfu/kungfu06.htm] [Consulta 2009, 03, 15].

[31] Zhengcai, L. El Misterio de la longevidad. Beijing: Ediciones en lenguas extranjeras; 1996.

[32] González, S. Las nueve perlas del chi kung (qigong). Camino de la longevidad. (2ª Ed.). Barcelona: Editorial Alas; 1996.

[33] Prouzet, A. La autocuración con el Qigong. Barcelona: Paidotribo; 2001. 